\title{
Low-Connectivity and Full-Coverage Three Dimensional Wireless Sensor Networks
}

\author{
Xiaole Bai \\ Dept. of Computer Science \\ and Engineering \\ The Ohio State University \\ Columbus, $\mathrm{OH}$, USA \\ baixia@cse.ohio- \\ state.edu
}

\author{
Chuanlin Zhang \\ Dept. of Mathematics \\ Jinan University \\ Guangzhou, P.R.CHINA \\ tclzhang@jnu.edu.cn
}

\author{
Dong Xuan \\ Dept. of Computer Science \\ and Engineering \\ The Ohio State University \\ Columbus, $\mathrm{OH}$, USA \\ xuan@cse.ohio-state.edu
}

\author{
Jin Teng \\ Dept. of Computer Science \\ City University of Hong Kong, \\ Hong Kong, P.R.CHINA \\ jinteng@cityu.edu.hk
}

\author{
Weijia Jia \\ Dept. of Computer Science \\ City University of Hong Kong, \\ Hong Kong, P.R.CHINA \\ wei.jia@ cityu.edu.hk
}

\begin{abstract}
Low-connectivity and full-coverage three dimensional Wireless Sensor Networks (WSNs) have many real-world applications. By low connectivity, we mean there are at least $k$ disjoint paths between any two sensor nodes in a WSN, where $k \leq 4$. In this paper, we design a set of patterns for these networks. In particular, we design and prove the optimality of 1- and 2-connectivity patterns under any value of the ratio of communication range $r_{c}$ over sensing range $r_{s}$, among regular lattice deployment patterns. We further propose a set of patterns to achieve 3 - and 4-connectivity patterns and investigate the evolutions among all the proposed low-connectivity patterns. Finally, we study the proposed patterns under several practical settings.
\end{abstract}

\section{Categories and Subject Descriptors}

C.2.1 [Computer-Communication networks]: Network Architecture and Design - network topology

\section{General Terms}

Theory

\section{Keywords}

Wireless sensor network topology, Optimal deployment pattern, Coverage, Connectivity

\section{INTRODUCTION}

In this paper, we study the problem of how to construct a three dimensional (3D) Wireless Sensor Network (WSN) that

Permission to make digital or hard copies of all or part of this work for personal or classroom use is granted without fee provided that copies are not made or distributed for profit or commercial advantage and that copies bear this notice and the full citation on the first page. To copy otherwise, to republish, to post on servers or to redistribute to lists, requires prior specific permission and/or a fee.

MobiHoc'09, May 18-21, 2009, New Orleans, Louisiana, USA.

Copyright 2009 ACM 978-1-60558-531-4/09/05 ...\$5.00. achieves low-connectivity and full-coverage by using the least number of sensors. By low connectivity, we mean there are at least $k$ disjoint paths between any two sensors, where $k \leq 4$.

\subsection{Motivation}

Our research on the above problem is motivated by the following two important facts.

- Low-connectivity and full-coverage three dimensional WSNs have many real-world applications: WSNs deployed in 3D aerial space can be used to support intelligent 3D vision systems [1], human paropsia [2], constructing aerial defense system [3], and aerosphere pollution monitoring [4] etc. 3D underwater WSNs also have various important applications [5] [6]. Sensor nodes have sensing capability, and in many applications, need to connect/ communicate with each other for data routing and aggregation. Low-connectivity WSNs are paticularly popular in real-world 3D WSN applications [5][6][7]. One of the important reasons is that 3D sensors are much more expensive than their $2 \mathrm{D}$ counterparts.

- The optimal deployment pattern problem is fundamental in 3D WSNs: Research towards solving this problem has both theoretical and practical significance. First, since today sensor nodes deployed in 3D aerial space and watery space are expensive, deploying the minimum necessary to achieve coverage and connectivity is important for economic reasons. Second, if the optimal deployment pattern has been known, it is then possible to compute, rather precisely, the efficiency of the commonly used patterns in 3D deployment in practice. Third, knowledge of optimal pattern can also provide guidance for designing topology control algorithms in 3D WSNs, and provide references for evaluating the performance of these algorithms.

\subsection{Related Work}

There are two sets of work related to the low-connectivity and full-coverage deployment problem in WSNs.

- Sphere Covering and Packing in Discrete Computational Geometry One closely related problem in discrete computational geometry is covering problem, especially sphere covering in 3D Euclidean space. In 1887, Lord Kelvin provided a 
conjectured answer to the problem of "What is the optimal way to fill a three dimensional space with cells of equal volume, so that the surface area (interface area) is minimum?". His answer states that the Voronoi polyhedrons in the optimal covering strategy are 14-sided truncated octahedrons [8]. To date, the proof for Kelvin's conjecture remains open. However, there are valuable efforts on the covering problem under certain conditions. One important condition is the spheres are placed following certain regularity. In [9], R. Bambah first proved that the least covering density of a 3D space by identical spheres is $5 \sqrt{5} \pi / 24$ (the definition of covering density will be given later). E. Barnes in [10] and L. Few in [11] proved the same result in different ways. Another closely related problem in discrete computational geometry is sphere packing in 3D Euclidean space. Sphere packing considers arrangements of non-overlapping identical spheres filling a space. There have been several works on the packing problem [14]. One of the most famous results is known as Kepler's conjecture. In 1611, Johannes Kepler conjectured the maximum possible density for sphere packing is $\pi / \sqrt{18}$. There is no rigorous proof until 2005. T. C. Hales in [15] accomplished the proof showing that no packing of identical spheres in 3D Euclidean space can have density greater than $\pi / \sqrt{18}$, which is the density of the face-centered cubic packing [15], [16], [17]. None of the above efforts considers connectivity in 3D Euclidean space.

- Connectivity and Coverage in WSN Deployment S. Alam and Z. Haas in [21] suggested the sensor deployment pattern that creates the Voronoi tessellation of truncated octahedral cells in 3D space. The suggestion is directly from Kelvin's conjecture. The numerical data in [21] illustrates truncated octahedron tessellation is better than the tessellations of cube, hexagonal prism, and rhombic dodecahedron. However, the optimality proof for truncated octahedron tessellation is untouched. Besides the efforts focused on the optimal deployment strategy, there are some works in 3D sensor deployment addressing other issues related to coverage. In [22], a deployment algorithm is proposed to "repair" coverage holes once they are discovered in a 3D volume. In [23], some sufficient conditions are presented for sensors in a given deployment to check if every points in a $3 \mathrm{D}$ volume is covered by at least $k$ sensors. None of these two provides any lead towards the optimality of deployment patterns in 3D WSNs. We notice there are many valuable efforts on 3D routing and localization, e.g., [24], [25], [7], [26], etc. Our research can act as a complement to them by providing new network topologies as a carrier for their algorithms and protocols. In 2D WSNs, X. Bai et al. in [18], [19], and [20] studied several optimal deployment patterns to achieve multiple connectivity and full coverage in $2 \mathrm{D}$ WSNs. The optimality of the proposed patterns in [20] is proved under the constraints of regularity. X. Bai et al. have made an attempt to further the exploration of optimal lattice patterns into three dimensional space, and some results on high connectivity are presented in [31].

\subsection{Our Contributions}

In this paper, we study the low-connectivity and full-coverage deployment problem in the domain of lattice. Our work is inspired by the efforts both in discrete computational geometry and WSNs. As discussed above, R. Bambah in [9] first presented some results under the regularity constraints brought by lattice (the definition of lattice will be given later). Then there were some follow-up efforts in the area [10], [11], [12]

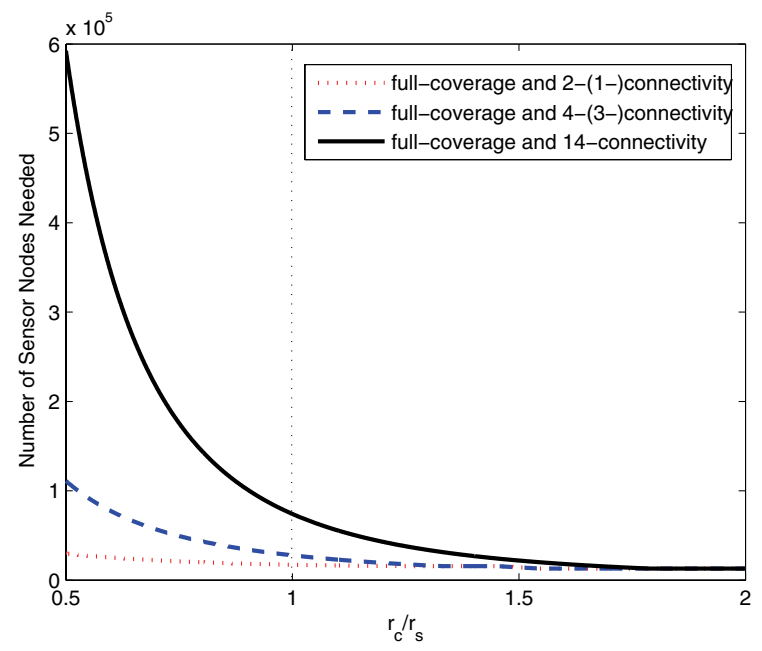

Figure 1: Sensor number needed for various $r_{c} / r_{s}$ to achieve 2- (1-), 4- (3-) and 14-connectivity by optimal lattice patterns, respectively. The $3 \mathrm{D}$ deployment space is $1,000^{3} \mathrm{~m}^{3}$. Sensing range $r_{s}$ is $30 \mathrm{~m}$ and communication range $r_{c}$ varies from $15 \mathrm{~m}$ to $60 \mathrm{~m}$.

and [13]. All these efforts only focus on coverage. In 2D WSNs, all the patterns X. Bai et al. proposed in [18], [19], [20] show the property of regularity. Among them, some have been proved to be optimal among all possible patterns [18], and some are proved to be optimal among the regular patterns [20]. The research interest for optimal patterns under certain regularity constraints has arisen because, besides pure theoretical intentions, it has been noticed that many important natural constructs show strong regularity in their constructing components. In 3D space, one of the most universal and important structure with the properties of periodicity and homogeneity is lattice. Typical examples include structures of numerous crystalline solids in our daily life, like coal, salt, ice, etc.

We highlight our contributions as follows.

- New deployment patterns with optimality: We have designed a set of patterns to construct 3D WSNs that achieve full coverage and k-connectivity where $k \leq 4$. We have proved the optimality of 1-connectivity and 2-connectivity patterns under any value of $r_{c} / r_{s}$ among regular lattice deployment patterns (defined in Section 2). Compared with the pattern proposed in [21] that achieves 14-connectivity, our patterns can save a great amount of nodes, as shown in Fig. 1. We observe when $r_{c} / r_{s}=1$, the number needed to achieve 14-connectivity is around 2.5 times that to achieve 3- or 4-connectivity, and 3.5 times that to achieve 1- or 2-connectivity. This number difference will increase as $r_{c} / r_{s}$ decreases. When $r_{c} / r_{s}=0.5$, the number needed to achieve 14-connectivity is almost 6 times that to achieve 3- or 4-connectivity, and around 18 times that to achieve 1- or 2-connectivity.

- Pattern evolutions: We have investigated the evolutions among all the proposed low-connectivity patterns. This study provides insights on exploration for optimal deployment patterns.

- Practical setting considerations: Above optimal patterns are based on the sphere sensing and communication models. As we know, to build up theoretical foundation, abstraction is inevitable. However, it is also important to study the pat- 
terns under more practical models. In the paper, we study the proposed patterns when practical models are considered. Besides, we also investigate an inherited problem among low connectivity networks, i.e. the long path problem [19], for the proposed deployment patterns.

The rest of paper is organized as follows. After presenting definition and notations in Section 2, we introduce 1- and 2connectivity patterns and prove their optimality in Section 3. In Section 4, we present 3- and 4-connectivity patterns and discuss pattern evolutions among all the proposed patterns. Practical considerations are studied in Section 5, followed by the conclusion of this paper.

\section{DEFINITIONS AND NOTATIONS}

Similar to the context for optimal deployment pattern research in 2D, we consider that all sensors are of same type and have sphere-shaped communication domain with radius $r_{c}$ and sensing field with radius $r_{s}$. The deployment region is considered vast enough such that its boundary can be ignored. We discuss in Section 5 practical considerations which are beyond these mathematical abstractions. In the following, we introduce some important definitions.

Definition 2.1. Right Parallelepiped, Axle Set, F-diagonal, B-diagonal: A hexahedron is called a right parallelepiped if its bases are parallelograms aligned one directly above the other and has lateral faces that are rectangles. Any three edges of a parallelepiped are called an axle set if any two of them are not parallel. The diagonals of the parallelepiped faces are called F-diagonals. The body diagonals of a parallelepiped are called B-diagonals.

Definition 2.2. Basic Lattice, Seed Parallelepiped: Given a right parallelepiped $\alpha$, the set $\Lambda$ is called a basic lattice generated by right parallelepiped $\alpha$ if $\Lambda$ is composed of all the vertices generated by shifting $\alpha$ to its three edges' directions with shift distance being integer times the corresponding edge length. This right parallelepiped $\alpha$ is called the seed parallelepiped for $\Lambda$.

For example, if $\alpha$ is a unit cube with edge length equal to 1 , we set its one vertex as the origin point and three lines passing through the axle set intersected at this vertex as the axes of a reference frame, then the basic lattice generated by $\alpha$ is the set of points with integer coordinates in this reference system.

It is worth noting that one basic lattice may have different seed parallelepipeds, but it is determined as long as one of them is given.

Definition 2.3. Body-Centered Lattice: Given a basic lattice $\Lambda^{\prime}$ generated by seed parallelepiped $\alpha$, point set $\Lambda$ is called a body-centered lattice if it is composed of all points in $\Lambda^{\prime}$ and all the center points of $\alpha$ in the process of generating $\Lambda^{\prime}$.

A body-centered lattice is called body-centered cubic lattice (bcc lattice in short) when its seed parallelepiped is a cube. Meanwhile a body-centered cuboid lattice will be generated if its seed parallelepiped is a cuboid.

In this paper, we study regular lattices. A regular lattice $\Lambda$ is either a basic lattice generated by its seed right parallelepiped $\alpha$ or a body-centered cuboid lattice generated by its seed cuboid $\alpha$.
Definition 2.4. Coverage Lattice with Radius r: Given lattice $\Lambda$ and spheres with radius $r$ centering at each point in $\Lambda, \Lambda$ is called a coverage lattice with radius $r$ if every point in a $3 D$ volume can be covered by at least one sphere.

DEFINITION 2.5. Lattice $\Lambda$ Pattern: Given sensors with sensing range $r_{s}$ and a lattice $\Lambda$, a sensor deployment scheme is called lattice $\Lambda$ pattern if sensors are deployed at each point in $\Lambda$ and $\Lambda$ is a coverage lattice with $r_{s}$.

From Definition 2.5, when the term "lattice pattern" is used in this paper, full coverage is always implied.

DEFINITION 2.6. Covering Density: If $\Lambda$ is a coverage lattice with radius $r$ and generated by seed parallelepiped $\alpha$, then the ratio of the total volume of the spheres with radius $r$ covering $\alpha$ to the volume of $\alpha$ is called covering density of $\Lambda$ with radius $r$, denoted by $\sigma(\Lambda, r)$.

Given a fixed $r_{s}$ and two lattices $\Lambda$ and $\Lambda^{\prime}$, if $\sigma\left(\Lambda, r_{s}\right)<$ $\sigma\left(\Lambda^{\prime}, r_{s}\right)$, then lattice $\Lambda$ pattern is better than lattice $\Lambda^{\prime}$ pattern since less sensor nodes are needed in lattices $\Lambda$ pattern to achieve full coverage.

Definition 2.7. Optimal Lattice Pattern: Given sensing $r$ ange $r_{s}$, a lattice $\Lambda$ pattern is called the optimal lattice pattern if $\sigma\left(\Lambda, r_{s}\right)$ is minimum among all regular lattice patterns.

Sensor deployment patterns in 3D WSNs are numberless and can be complicated. As we mentioned in Section 1, to find and prove the optimal deployment patterns to fully cover a 3D space among all possible patterns is very hard even when the connectivity is not considered. It has been noticed that many important natural constructs in 3D space show strong periodicity and homogeneity in their constructing components. One of the most universal and important structure with such properties is lattice. In this paper, we are to explore the optimal patterns among regular lattice patterns.

\section{LATTICE PATTERN FOR 1- AND 2-CONNECTIVITY}

In this section, we first present optimal lattice patterns that achieve 1- and 2-connectivity, and then the optimality proof.

Due to their symmetry, lattice patterns with exactly odd connectivity do not exist. We only need to consider those that achieve even connectivity. Naturally, the optimal lattice patterns that achieve 1-connectivity are optimal ones that achieve 2-connectivity.

\subsection{Pattern Description}

The proposed lattice patterns for 1- or 2-connectivity in 3D space are shown in Fig. 2.

- When $r_{c} / r_{s}<4 / 3$, the pattern follows a body-centered lattice, denoted by $\Lambda_{2-1}$, which is generated by a cuboid $\alpha$ with upper and bottom faces each with edge length $e_{1}=$ $\sqrt{\left(3 r_{s}^{2}-r_{c}^{2}+r_{s} \sqrt{9 r_{s}^{2}-2 r_{c}^{2}}\right) / 2}, e_{2}=\left(3 r_{s}+\sqrt{9 r_{s}^{2}-2 r_{c}^{2}}\right) / 2$ and its center. The height of $\alpha$ is $r_{c}$. This seed cuboid $\alpha$ and its center are illustrated by ABCDEFGH and I in Fig. 2(a1). Any sensor is able to connect with its two neighbors along the direction of height, as illustrated by sensor A in Fig. 2(a1).

- When $4 / 3 \leq r_{c} / r_{s}<12 / \sqrt{9+32 \sqrt{3}}$, the pattern follows a body-centered lattice, denoted by $\Lambda_{2-2}$, which is generated by a cuboid $\alpha$ with upper and bottom faces each with 


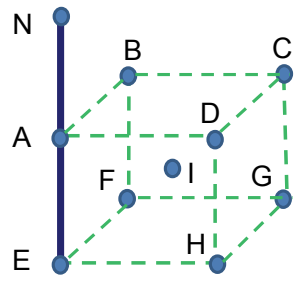

(a1)

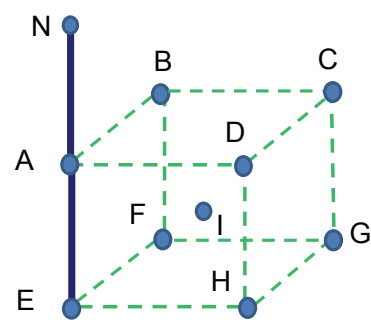

(a2)

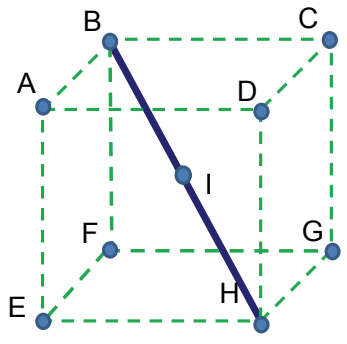

(a3)

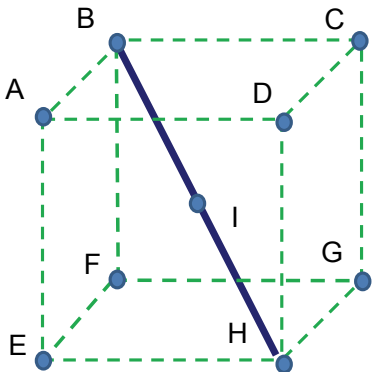

(a4)

Figure 2: Lattice patterns that achieves 1- or 2-connectivity and full coverage. The dashed lines illustrate how lattices are constructed. The connection links are shown in bold solid lines. (a1) Body-centered lattice $\Lambda_{2-1}$ pattern when $r_{c} / r_{s}<4 / 3$. (a2) Body-centered lattice $\Lambda_{2-2}$ pattern when $4 / 3 \leq r_{c} / r_{s}<12 / \sqrt{9+32 \sqrt{3}}$. (a3) Body-centered lattice $\Lambda_{2-3}$ pattern when $12 / \sqrt{9+32 \sqrt{3}} \leq r_{c} / r_{s}<2 \sqrt{3} / \sqrt{5}$. (a4) Body-centered lattice $\Lambda_{2-4}$ pattern when $2 \sqrt{3} / \sqrt{5} \leq r_{c} / r_{s}$.

edge length $e_{3}=e_{4}=\sqrt{4 r_{s}^{2}-r_{c}^{2} / 4}$ and its center. The height of $\alpha$ is $r_{c}$. This seed cuboid $\alpha$ and its center are illustrated by ABCDEFGH and I in Fig. 2(a2). Any sensor can connect with its two neighbors as illustrated by sensor $\mathrm{A}$.

- When $12 / \sqrt{9+32 \sqrt{3}} \leq r_{c} / r_{s}<2 \sqrt{3} / \sqrt{5}$, the pattern follows a body-centered lattice, denoted by $\Lambda_{2-3}$, which is generated by a cube $\alpha$ with edge length $e_{5}=2 r_{c} / \sqrt{3}$ and its center. This seed cube and its center is illustrated by ABCDEFGH and I in Fig. 2(a3). Any sensor is able to connect with its two neighbors along the direction of B-diagonal, as illustrated by sensor I in Fig. 2(a3).

- When $2 \sqrt{3} / \sqrt{5} \leq r_{c} / r_{s}$, the pattern also follows a bodycentered lattice, denoted by $\Lambda_{2-4}$, which is generated by a cube $\alpha$ with edge length $e_{6}=4 r_{s} / \sqrt{5}$ and its center. This seed cube and its center is illustrated by ABCDEFGH and I in Fig. 2(a4). Any sensor is able to connect with its two neighbors along the direction of B-diagonal, as illustrated by sensor I in Fig. 2(a4).

We note that some extra nodes are needed at the boundaries of 3D deployment volume for global connectivity when lattice $\Lambda_{2-1}$ or $\Lambda_{2-2}$ patterns are used. More discussions on this issue are provided in Section 5.

THEOREM 3.1. To achieve 1- or 2-connectivity and full coverage in $3 D$ space, the body-centered lattice $\Lambda_{2-1}$ pattern is an optimal regular lattice pattern when $r_{c} / r_{s}<4 / 3$, the bodycentered lattice $\Lambda_{2-2}$ pattern is an optimal regular lattice pattern when $4 / 3 \leq r_{c} / r_{s}<12 / \sqrt{9+32 \sqrt{3}}$, the body-centered lattice $\Lambda_{2-3}$ pattern is an optimal regular lattice pattern when $12 / \sqrt{9+32 \sqrt{3}} \leq r_{c} / r_{s}<2 \sqrt{3} / \sqrt{5}$, and the body-centered lattice $\Lambda_{2-4}$ pattern is an optimal regular lattice pattern when $2 \sqrt{3} / \sqrt{5} \leq r_{c} / r_{s}$.

\subsection{Optimality Proof}

In this section, we first present the proof road map for Theorem 3.1. We then present the proof details followed by remarks.

- Road map From Definition 2.7, to prove optimality is equivalent to find the lattice $\Lambda$ pattern with the least covering density $\sigma(\Lambda, r)$, which is denoted by $\sigma_{M I N}$. To get $\sigma_{M I N}$, we need to consider all regular basic lattice patterns as well as regular body-centered lattice patterns, obtain $\sigma_{M I N}^{\prime}$ 's for all cases, and then compare them.

Covering density is $4 \pi r_{s}^{3} /(3 V)$ for basic lattice patterns, and is $8 \pi r_{s}^{3} /(3 V)$ for body-centered lattice patterns, where $V$ is the volume of the seed parallelepiped. To obtain $\sigma_{M I N}^{\prime}$ for each case, we are to obtain the maximum volume, which is denoted by $V_{M A X}^{\prime}$, for each case, as is shown in equation (1), where $x, y$ and $z$ are the lengths of three non-parallel edges of the right parallelepiped and $\gamma$ is the included angle of the bottom parallelogram.

$$
\max f(x, y, z, \gamma)=x y z \sin \gamma .
$$

$V_{M A X}^{\prime}$ can be obtained by solving a nonlinear optimization problem (1) under constraints generated from full coverage and desirable connectivity. In the following, we present proof details.

- Proof Both coverage constraints and connectivity constraints are considered explicitly for (1). Coverage constraints are reflected by first properly choosing a certain face or a certain geometry point or both of the seed parallelepiped and then letting them be covered. This dimensionality reduction is important since it decides the number of constraints for the nonlinear optimization and thus decides the feasibility of solving it. Connectivity constraints are reflected by lengths of different set of edges, or F-diagonals, or B-diagonals of the seed parallelepiped.

Since constraints are different for basic lattice pattern case and for body-centered lattice pattern case. In the following part, we show how to obtain these constraints for basic lattices and body-centered lattice, respectively.

Case A. Basic Lattice We consider coverage constraints first, then connectivity constraints.

We denote the constraints for satisfying full coverage in this case by $\operatorname{Cov}-B L$. As shown in Fig. 3 (a), we denote the parallelogram that is parallel to the bottom at $z / 2$ in a seed parallelepiped by $\Omega_{z / 2}$. Covering $\Omega_{z / 2}$ is a necessary condition for full coverage. Now we show it is sufficient. Compared with other parallelograms parallel to $\Omega_{z / 2}, \Omega_{z / 2}$ is the hardest to cover since the intersections of sensing spheres on this plane (intersections are disks) are smaller than those on other parallelograms. If the parallelogram $\Omega_{z / 2}$ is covered, then any other parallelogram parallel to $\Omega_{z / 2}$ must be covered by larger intersection disks. Then we can transform the 


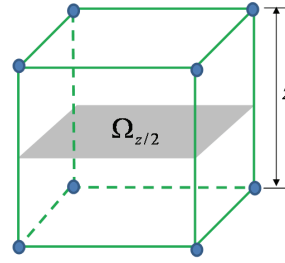

(a)

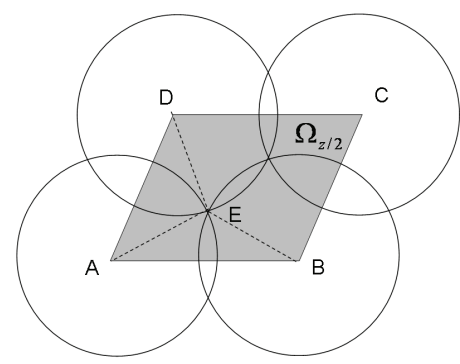

(b)
Figure 3: (a)The parallelogram at the middle of height $z$ is denoted by $\Omega_{z / 2}$. (b) The most efficient way to cover the middle parallelogram at $z / 2$.

constraints to fully cover the seed parallelepiped to the constraints to fully cove $\Omega_{z / 2}$.

The most efficient way to cover $\Omega_{z / 2}$ is to let the overlapped area of any three disks (intersections of sensing spheres by the plane) be zero, as illustrated in Fig. 3(b). Let $E$ be the excenter of the triangle $A B D$ (with 3 acute angles, implying $\angle D A B=\gamma \leq \pi / 2)$. Then distance between the ex-center to any of the three vertices is the smallest disk radius we can have. Denote this radius by $r^{\prime}$. Assume $A$ is the origin point $o$ at $(0,0), B$ at $(x, 0)$ and $D$ at $(y \cos \gamma, y \sin \gamma)$, then we can get coordinates of $E$, i.e., $(x / 2,(y-x \cos \gamma) /(2 y))$. We then have $r^{\prime}=\sqrt{y^{2}+x^{2}-2 x y \cos \gamma} / 2 \sin \gamma$. This is the smallest radius ever possible to cover the whole seed parallelepiped. Hence, we get the condition $\operatorname{Cov}-B L$ as follows, $\sqrt{r_{s}^{2}-z^{2} / 4} \geq$ $\sqrt{y^{2}+x^{2}-2 x y \cos \gamma} /(2 \sin \gamma)$, which can be rewritten as

$$
x^{2}+y^{2}-2 x y \cos \gamma \leq\left(4 r_{s}^{2}-z^{2}\right) \sin ^{2} \gamma .
$$

Now we consider the constraints for connectivity. The connection edge can be either one edge of the seed parallelepiped, or a F-diagonal, or a B-diagonal. Note that in basic lattice, if a F-diagonal or a B-diagonal is the connection edge, then at least two edges of the base are also be the connection edge. Hence, we only need to consider two cases here, namely, $x \leq r_{c}$ or $z \leq r_{c}$. Denote these two connectivity constraint by Con-BL-1 and Con-BL-2.

The constraints of $C o v-B L$ AND Con- $B L-1$ then can be written as

$$
\left\{\begin{array}{l}
x^{2}+y^{2}-2 x y \cos \gamma \leq\left(4 r_{s}^{2}-z^{2}\right) \sin ^{2} \gamma \\
x \leq r_{c}
\end{array}\right.
$$

Then we have solution as follows.

- When $r_{c} / r_{s} \leq \sqrt{2}, x=r_{c}, y=\sqrt{2} r_{s}, z=2 r_{s} \sqrt{\frac{4 r_{s}^{2}-r_{c}^{2}}{8 r_{s}^{2}-r_{c}^{2}}}$ and $\gamma=\arccos \left(r_{c} / 2 \sqrt{2} r_{s}\right), V_{M A X-1}^{\prime}=r_{c} r_{s} \sqrt{4 r_{s}^{2}-r_{c}^{2}}$ and $\sigma_{M I N-1}^{\prime}=4 \pi r_{s}^{2} /\left(3 r_{c} \sqrt{4 r_{s}^{2}-r_{c}^{2}}\right)$.

- When $\sqrt{2} \leq r_{c} / r_{s} \leq 4 / \sqrt{5}, x=y=\sqrt{2} r_{s}, z=2 r_{s} / \sqrt{3}$ and $\gamma=\pi / 3, V_{M A X-1}^{\prime}=2 r_{s}^{3}$ and $\sigma_{M I N-1}^{\prime}=2 \pi / 3$.

The constraints of $C o v-B L$ AND Con-BL-2 then can be written as

$$
\left\{\begin{array}{l}
x^{2}+y^{2}-2 x y \cos \gamma \leq\left(4 r_{s}^{2}-z^{2}\right) \sin ^{2} \gamma \\
z \leq r_{c}
\end{array}\right.
$$

We have solution as follows.

- When $r_{c} / r_{s} \leq 2 / \sqrt{3}, x=y=\sqrt{3} \sqrt{4 r_{s}^{2}-r_{c}^{2}}, z=r_{c}$ and $\gamma=\pi / 3, V_{M A X-2}^{\prime}=3 r_{c} \sqrt{3}\left(4 r_{s}^{2}-r_{c}^{2}\right) / 8$ and $\sigma_{M I N-2}^{\prime}=$

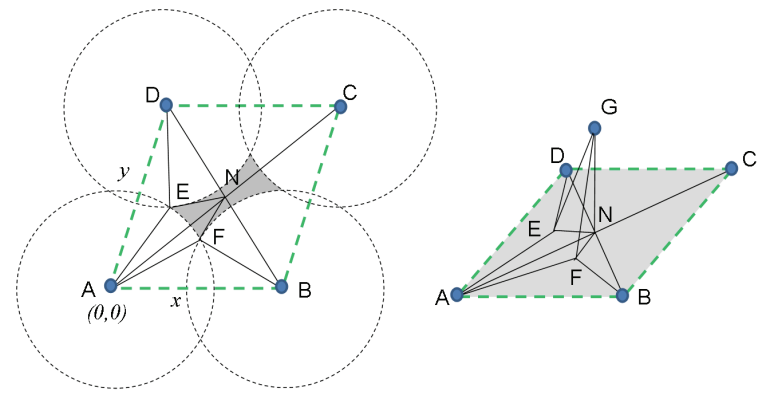

(a)

(b)

Figure 4: (a) The area in base ABCD that is not covered by four sensors at base vertices is indicated by shaded area. (b) The uncovered area should be covered by the sensor $G$ that is at the center. $|G N|=z / 2$.

$32 \pi r_{s}^{3} /\left(9 r_{c} \sqrt{3}\left(4 r_{s}^{2}-r_{c}^{2}\right)\right)$.

- When $2 / \sqrt{3} \leq r_{c} / r_{s} \leq 4 / \sqrt{5}, x=y=\sqrt{2} r_{s}, z=2 r_{s} / \sqrt{3}$ and $\gamma=\pi / 3, V_{M A X-2}^{\prime}=2 r_{s}^{3}$ and $\sigma_{M I N-2}^{\prime}=2 \pi / 3$.

We note it is not necessary to consider the range $r_{c} / r_{s} \geq$ $4 / \sqrt{5}$ since the pattern proposed in [21] from Kevin's conjecture can achieve 14-connectivity in this range.

Case B. Body-Centered Lattice We consider coverage constraints first, then connectivity constraints. Though we mainly study body-centered cuboid lattice, yet we'll derive constraints for more general lattices, i.e. body-centered lattices generated by right parallelepiped. These results will be helpful to further efforts in the optimality computation.

Denote the coverage constraints from this case by Cov-BCL. Among three edges, $x, y$ and $z$, at most one of them can be larger than $2 r_{s}$. Otherwise, the face with edges that are both larger than $2 r_{s}$ can never be covered. Hence, we have three cases to consider as follows. First, no edge is longer than $2 r_{s}$. Denote the coverage constraints from this case by CovBCL-1. Second, one edge of base (at plan xoy) is longer than $2 r_{s}$. Without loss of generality, we can assume $x \geq 2 r_{s}$. The corresponding coverage constraints are denoted by Cov-BCL2. Third, the height of seed parallelepiped, $z$, is longer than $2 r_{s}$. The constraints are denoted by Cov-BCL-3.

We now consider the first case where there is no edge is longer than $2 r_{s}$. A sufficient and necessary condition for full coverage of body-centered lattice is that all faces of the seed parallelepiped are covered. We start from the base. On the xOy plane, as illustrated in Fig. 4(a), we have $|A E|=|D E|=$ $|A F|=|B F|=r_{s}, \angle D A B=\gamma$. We have both $|E N|$ and $|F N|$ should be smaller than $\sqrt{r_{s}^{2}-z^{2} / 4}$ to ensure the uncovered area should be covered by the sensor $G$ at the center, as illustrated in Fig. 4(b). We have the following constraints (5).

$$
\left\{\begin{array}{l}
y^{2}+z^{2}-x^{2} \leq 2 y \sin \gamma \sqrt{4 r_{s}^{2}-x^{2}} \\
x^{2}+z^{2}-y^{2} \leq 2 x \sin \gamma \sqrt{4 r_{s}^{2}-y^{2}}
\end{array}\right.
$$

Now we study the face on plane $x o z$, as illustrated in Fig. 5, we have $|A E|=|J E|=|J F|=|K F|=r_{s}$ and sensor $G$ locates at the center, and $\mathrm{M}$ is its projection on the side face ABJK. To ensure the area that is not covered by the four sensors at face vertices should be covered by the sensor G, we 


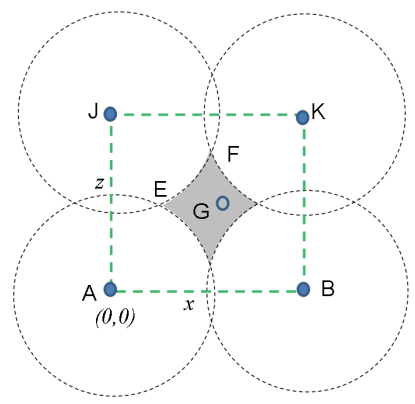

(a)

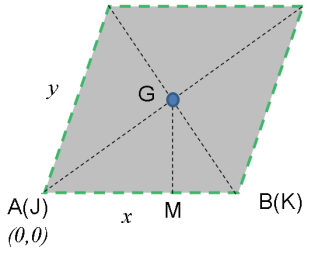

(b)
Figure 5: (a) The area in a side face ABKJ that is not covered by the four sensors at base vertices is indicated by shaded area. (b) The uncovered area should be covered by the sensor $G$ that is at the center. $\mathrm{M}$ is the projection of $\mathrm{G}$ at the side face.

have following condition

$$
|M E| \leq \sqrt{r_{s}^{2}-\frac{y^{2} \sin ^{2} \gamma}{4}}, \quad|M F| \leq \sqrt{r_{s}^{2}-\frac{y^{2} \sin ^{2} \gamma}{4}} .
$$

That is,

$$
\left\{\begin{array}{l}
x+y \cos \gamma-\sqrt{4 r_{s}^{2}-z^{2}}-\sqrt{4 r_{s}^{2}-y^{2} \sin ^{2} \gamma} \leq 0 \\
y^{2}+z^{2}-x^{2}-2 z \sqrt{4 r_{s}^{2}-x^{2}} \leq 0
\end{array}\right.
$$

By exchange $x$ with $y$, we can have the constraints for the face at plan yoz as follows.

$$
\left\{\begin{array}{l}
y+x \cos \gamma-\sqrt{4 r_{s}^{2}-z^{2}}-\sqrt{4 r_{s}^{2}-x^{2} \sin ^{2} \gamma} \leq 0 \\
x^{2}+z^{2}-y^{2}-2 z \sqrt{4 r_{s}^{2}-y^{2}} \leq 0
\end{array}\right.
$$

Combine (5), (6) and (7), we obtain constraints Cov-BCL-1 as follows

$$
\left\{\begin{array}{l}
y^{2}+z^{2}-x^{2} \leq 2 y \sin \gamma \sqrt{4 r_{s}^{2}-x^{2}} \\
x^{2}+z^{2}-y^{2} \leq 2 x \sin \gamma \sqrt{4 r_{s}^{2}-y^{2}} \\
x+y \cos \gamma-\sqrt{4 r_{s}^{2}-z^{2}}-\sqrt{4 r_{s}^{2}-y^{2} \sin ^{2} \gamma} \leq 0 \\
y+x \cos \gamma-\sqrt{4 r_{s}^{2}-z^{2}}-\sqrt{4 r_{s}^{2}-x^{2} \sin ^{2} \gamma} \leq 0 \\
y^{2}+z^{2}-x^{2}-2 z \sqrt{4 r_{s}^{2}-x^{2}} \leq 0 \\
x^{2}+z^{2}-y^{2}-2 z \sqrt{4 r_{s}^{2}-y^{2}} \leq 0 \\
x \leq 2 r_{s}, y \leq 2 r_{s}, z \leq 2 r_{s} \\
0 \leq \gamma \leq \frac{\pi}{2}
\end{array}\right.
$$

Similarly, we can obtain the constraints Cov-BCL-2 and CovBCL-3 for the second case and third case respectively. Con- straints Cov-BCL-2 are

$$
\left\{\begin{array}{l}
x^{2}+y^{2}-z^{2}+2 x y \cos \gamma \leq 2(y+x \cos \gamma) \sqrt{4 r_{s}^{2}-z^{2}} \\
x^{2}+y^{2}-z^{2}-2 x y \cos \gamma \leq 2(y-x \cos \gamma) \sqrt{4 r_{s}^{2}-z^{2}} \\
x^{2}+z^{2}-y^{2} \leq 2 z \sqrt{4 r_{s}^{2}-y^{2}} \\
x^{2}+y^{2}+z^{2}+2 x y \cos \gamma \leq 4 r_{s}(x+y \cos \gamma) \\
x^{2}+y^{2}+z^{2}-2 x y \cos \gamma \leq 4 r_{s}(x-y \cos \gamma) \\
x \geq 2 r_{s}, y \leq 2 r_{s}, z \leq 2 r_{s} \\
0 \leq \gamma \leq \frac{\pi}{2}
\end{array}\right.
$$

And Constraints Cov-BCL-3 are

$$
\left\{\begin{array}{l}
x^{2}+y^{2}-2 x y \cos \gamma \leq 4 r_{s}^{2} \sin ^{2} \gamma \\
x^{2}+y^{2}+z^{2}+2 x y \cos \gamma \leq 4 r_{s} z \\
x^{2}+z^{2}-y^{2} \leq 2 z \sqrt{4 r_{s}^{2}-y^{2}} \\
y^{2}+z^{2}-x^{2} \leq 2 z \sqrt{4 r_{s}^{2}-y^{2}} \\
x \leq 2 r_{s}, y \leq 2 r_{s}, z \geq 2 r_{s} \\
0 \leq \gamma \leq \frac{\pi}{2}
\end{array}\right.
$$

Now we consider the constraints for connectivity requirements. We'll focus on the regular lattice patterns as mentioned in Section 2. With body-centered cuboid lattices, we need to consider three cases. First, one edge, $x$ or $y$, is the connection edge. We denote the constraints for this case by Con-BCL-1. Second, the height, $z$, is the connection edge. The constraints for this case is denoted by Con-BCL-2. Third, the half of B-diagonal is the connection edge. We have the constraints $x^{2}+y^{2}+z^{2}-2 x y \cos \gamma \leq 4 r_{c}^{2}$. The constraints for this case is denoted by Con-BCL-3.

To obtain the optimal deployment pattern for body centered lattice, we are to solve (1) under following constraints cases.

Case B.1. Constraints can be expressed as (Cov-BCL-1 OR Cov-BCL-2 OR Cov-BCL-3)AND Con-BCL-1.

We obtain the solution for this case as follows.

- When $4 / \sqrt{5} \leq r_{c} / r_{s}$, and $x=y=z=4 / \sqrt{5} r_{s}$, and $\gamma=$ $\pi / 2$, the seed parallelepiped takes maximal volume $V_{M A X-3}^{\prime}=$ $64 r_{s}^{3} / 5 \sqrt{5}$ and the corresponding converge density $\sigma_{M I N-3}^{\prime}=$ $5 \sqrt{5} \pi / 24$.

- When $4 / 3 \leq r_{c} / r_{s}<4 / \sqrt{5}$, and $x=r_{c}, y=z=$ $\sqrt{4 r_{s}^{2}-r_{c}^{2} / 4}$, and $\gamma=\pi / 2$, the seed parallelepiped takes maximal volume $V_{M A X-3}^{\prime}=r_{c}\left(4 r_{s}^{2}-r_{c}^{2} / 4\right)$ and the corresponding converge density $\sigma_{M I N-3}^{\prime}=32 \pi r_{s}^{3} / 3 r_{c}\left(16 r_{s}^{2}-r_{c}^{2}\right)$.

- When $r_{c} / r_{s}<4 / 3$, and $x=r_{c}$,

$y=\sqrt{\left(3 r_{s}^{2}-r_{c}^{2}+r_{s} \sqrt{9 r_{s}^{2}-2 r_{c}^{2}}\right) / 2}, z=\left(3 r_{s}+\sqrt{9 r_{s}^{2}-2 r_{c}^{2}}\right) / 2$, and $\gamma=\pi / 2$,the seed parallelepiped takes maximal volume $V_{M A X-3}^{\prime}$ as

$$
r_{c} \sqrt{\frac{3 r_{s}^{2}-r_{c}^{2}+r_{s} \sqrt{9 r_{s}^{2}-2 r_{c}^{2}}}{8}}\left(3 r_{s}+\sqrt{9 r_{s}^{2}-2 r_{c}^{2}}\right),
$$

and the corresponding converge density $\sigma_{M I N-3}^{\prime}$ is

$$
\frac{16 \sqrt{2} \pi r_{s}^{3}}{3 r_{c}\left(3 r_{s}+\sqrt{9 r_{s}^{2}-2 r_{c}^{2}}\right) \sqrt{\left(3 r_{s}^{2}-r_{c}^{2}+r_{s} \sqrt{9 r_{s}^{2}-2 r_{c}^{2}}\right)}} .
$$

Case B.2. Constraints can be expressed as (Cov-BCL-1 OR Cov-BCL-2 OR Cov-BCL-3)AND Con-BCL-2. 
The solution of Case B.2. is the same as that of Case B.1., since $x, y$ and $z$ are symmetrical when $\gamma=\pi / 2$.

Case B.3. Constraints can be expressed as (Cov-BCL-1 OR Cov-BCL-2 OR Cov-BCL-3)AND Con-BCL-3.

- When $2 \sqrt{3} / \sqrt{5} \leq r_{c} / r_{s}$, and $x=y=z=4 r_{s} / \sqrt{5}$, and $\gamma=\pi / 2$, the seed parallelepiped takes maximal volume $V_{M A X-4}^{\prime}=64 r_{s}^{3} / 5 \sqrt{5}$ and the corresponding converge density $\sigma_{M I N-4}^{\prime}=5 \sqrt{5} \pi / 24$.

- When $2 \sqrt{3} / \sqrt{5}>r_{c} / r_{s}$, and $x=y=z=2 r_{c} / \sqrt{3}$, and $\gamma=\pi / 2$, the seed parallelepiped takes maximal volume $V_{M A X-4}^{\prime}=8 r_{c}^{3} / 3 \sqrt{3}$ and the corresponding converge density $\sigma_{M I N-4}^{\prime}=\sqrt{3} \pi r_{s}^{3} / r_{c}^{3}$.

We are now ready to get the optimal lattice pattern with the smallest covering density $\sigma_{M I N}$ by comparing $\sigma_{M I N-1}^{\prime}$ and $\sigma_{M I N-2}^{\prime}$, as well as $\sigma_{M I N-3}^{\prime}, \sigma_{M I N-4}^{\prime}, \sigma_{M I N-5}^{\prime}$ in the interval of $r_{c} / r_{s}<4 / \sqrt{5}$. We have,

$$
\sigma_{M I N}= \begin{cases}\sigma_{M I N-3}^{\prime}, & r_{c} / r_{s}<12 \sqrt{9+32 \sqrt{3}} \\ \sigma_{M I N-4}^{\prime}, & 12 \sqrt{9+32 \sqrt{3}} \leq r_{c} / r_{s} \leq 4 / \sqrt{5}\end{cases}
$$

This concludes the proof for Theorem 3.1.

- Remarks Solving above nonlinear optimization problems is not trivial. The difficulty lies on the number of constraints and their complicated expressions. These constraints are obtained from coverage and connectivity requirements in lattice patterns generated by right parallelepiped $\alpha$ or cuboid $\beta$. However, when general lattice patterns are considered, coverage constraints are difficult to get and they have more complicated expressions. To solve nonlinear optimization problems for such cases is hard. We conjecture that our proposed patterns here are also optimal among general lattice patterns. Its proof is our on-going work.

\section{LATTICE PATTERN FOR 3- AND 4-CONNECTIVITY}

In this section, we present lattice patterns to achieve 3- and 4-connectivity. Similarly, optimal 4-connectivity lattice patterns are also optimal 3-connectivity lattice patterns.

\subsection{Pattern Description}

The optimal lattice pattern that achieves 3- or 4-connectivity is shown in Fig. 6.

- When $r_{c} / r_{s}<4 / 3$, the pattern follows a body-centered lattice, denoted by $\Lambda_{4-1}$, which is generated by a cuboid $\alpha$ with upper and bottom faces each with edge length $e_{7}=r_{c}$ and $e_{8}=2 r_{s}+\sqrt{4 r_{s}^{2}-2 r_{c}^{2}}$. The height of $\alpha$ is $r_{c}$. This seed cuboid and its center is illustrated by ABCDEFGH and I in Fig. 6(b1). Any sensor is able to connect with its four neighbors in one plane. For example, in Fig. 6(b1) sensor E is connected with $\mathrm{A}, \mathrm{N}, \mathrm{M}$ and $\mathrm{H}$.

- When $4 / 3 \leq r_{c} / r_{s}<2 \sqrt[3]{2} / \sqrt{3}$, the pattern also follows a body-centered lattice, denoted by $\Lambda_{4-2}$, which is generated by a cuboid $\alpha$ with upper and bottom faces each with edge length $e_{9}=4 r_{s} / 3$ and $e_{10}=8 r_{s} / 3$. The height of $\alpha$ is $4 r_{s} / 3$. This seed cuboid and its center is illustrated by ABCDEFGH and I in Fig. 6(b2). Any sensor is able to connect with its four neighbors in one plane. For example, in Fig. 6(b2) sensor E is connected with $\mathrm{A}, \mathrm{N}, \mathrm{M}$ and $\mathrm{H}$.
- When $2 \sqrt[3]{2} / \sqrt{3} \leq r_{c} / r_{s} \leq 2 \sqrt{3} / \sqrt{5}$, the pattern follows a body-centered lattice, denoted by $\Lambda_{4-3}$, which is generated by a cube $\alpha$ with edge length $e_{11}=2 r_{c} / \sqrt{3}$ and its center. This seed cube and its center is illustrated by ABCDEFGH and I in Fig. 6(b3). Any sensor is able to connect with its two neighbors along the direction of B-diagonals. For example, in Fig. 6(b3) sensor I is connected with A, B, G and $\mathrm{H}$.

- When $2 \sqrt{3} / \sqrt{5} \leq r_{c} / r_{s}$, the pattern also follows a bodycentered lattice, denoted by $\Lambda_{4-4}$, which is generated by a cube $\alpha$ with edge length $e_{12}=4 r_{s} / \sqrt{5}$ and its center. This seed cube and its center is illustrated by ABCDEFGH and I in Fig. 6(b4). Any sensor is able to connect with its two neighbors along the direction of B-diagonals. For example, in Fig. 6(b3) sensor I is connected with A, B, G and H.

We notice that some nodes are needed to add at the boundaries of 3D deployment volume to achieve global connectivity when lattice $\Lambda_{4-1}$ or $\Lambda_{4-2}$ patterns are adopted. More discussion on this issue are provided in Section 5.

\subsection{Pattern Evolution}

It is interesting and important to study how different patterns are related and how they evolve.

We note that from symmetry, the lattice $\Lambda_{2-3}$ pattern shown in Fig. 2(a3) actually achieve 8-connectivity (sensor I can connect with sensors $A, B, C, D, E, F, G$, and $H$ ), and the lattice $\Lambda_{2-4}$ pattern shown in Fig. 2(a4) actually achieve 14connectivity (sensor I can connect with sensors A, B, C, D, E, F, G, $\mathrm{H}$ as well as the sensors at centers of 6 neighboring cubes). We also note that, the lattice $\Lambda_{4-3}$ pattern shown in Fig. 2(b3) actually achieve 8-connectivity (sensor I can connect with sensors $A, B, C, D, E, F, G$, and $H$ ), and the lattice $\Lambda_{4-4}$ pattern shown in Fig. 2(b4) actually achieve 14connectivity (sensor I can connect with sensors A, B, C, D, E, F, G, H as well as the sensors at centers of 6 neighboring cubes).

Since a network with high connectivity also implies low connectivity, given a lattice $\Lambda$ pattern is an optimal lattice pattern to achieve $k_{1}$-connectivity and it actually achieves $k_{2}$-connectivity $\left(k_{2}>k_{1}\right)$, it will also be an optimal lattice pattern to achieve $k_{2}$-connectivity. Hence, we have the optimality of partial patterns we proposed for 4-connectivity as follows. To achieve 4-connectivity and full coverage in 3D space, the lattice $\Lambda_{4-3}$ pattern is an optimal lattice pattern when $2 \sqrt[3]{2} / \sqrt{3} \leq r_{c} / r_{s}<2 \sqrt{3} / \sqrt{5}$, and the lattice $\Lambda_{4-4}$ pattern is an optimal lattice pattern when $2 \sqrt{3} / \sqrt{5} \leq r_{c} / r_{s}$.

We only conjecture that the lattice $\Lambda_{4-1}$ pattern is an optimal lattice pattern when $r_{c} / r_{s}<4 / 3$ and the lattice $\Lambda_{4-2}$ pattern is an optimal lattice pattern when $4 / 3 \leq r_{c} / r_{s}<$ $2 \sqrt[3]{2} / \sqrt{3}$ in this paper. The lattice $\Lambda_{4-1}$ and $\Lambda_{4-2}$ patterns come from exhausting search and we are not aware any other lattice patterns with smaller covering density. The difficulty of directly proving optimality for 4-connectivity patterns lies on its various ways to achieve connectivity. Furthermore, we believe 1- and 2-connectivity are more fundamental and representative when low-connectivity is considered.

A close look at lattice pattern $\Lambda_{2-1}, \Lambda_{2-2}, \Lambda_{4-2}, \Lambda_{2-3}\left(\Lambda_{4-3}\right)$ and $\Lambda_{2-4}\left(\Lambda_{4-4}\right)$ reveals how patterns evolve as $r_{c} / r_{s}$ increases. In lattice $\Lambda_{2-1}, \Lambda_{2-2}$ or $\Lambda_{4-2}$ patterns, connectivity follows "lines" or "planes". The "lines" and "planes" are separate from each other, hence extra sensors are needed to add at volume boundaries to assure global connectivity. In lattice $\Lambda_{2-3}$ and $\Lambda_{2-4}$ pattern, where one sensor can connect to 8 or 14 neighbors, connectivity follows "bodies" and no extra sen- 


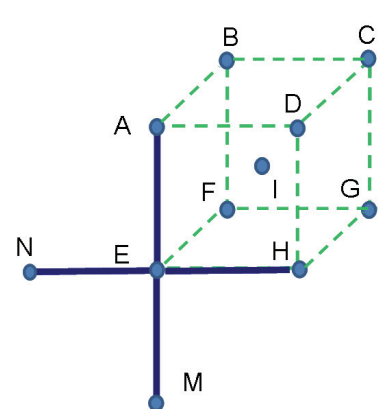

(b1)

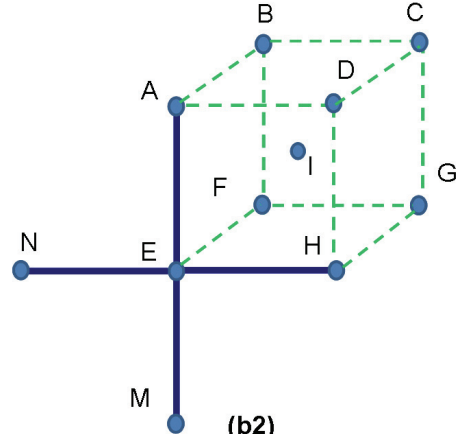

(b2)

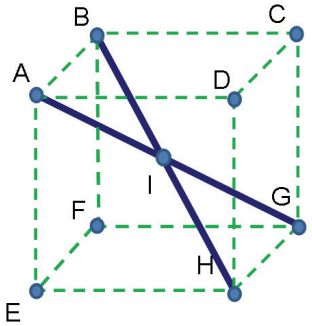

(b3)

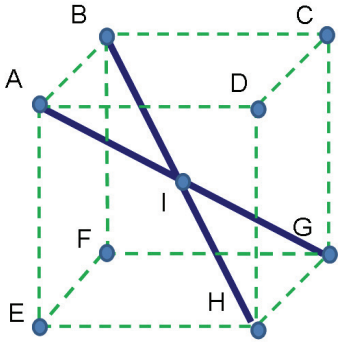

(b4)

Figure 6: Lattice patterns that achieves 3- or 4-connectivity and full coverage. The dashed lines illustrate how lattices are constructed. The connection links are shown in bold solid lines. (b1) Body-centered lattice $\Lambda_{4-1}$ pattern when $r_{c} / r_{s}<4 / 3$. (b2) Body-centered lattice $\Lambda_{4-2}$ pattern when $4 / 3 \leq r_{c} / r_{s}<2 \sqrt[3]{2} / \sqrt{3}$. (b3) Body-centered lattice $\Lambda_{4-3}$ pattern when $2 \sqrt[3]{2} / \sqrt{3} \leq r_{c} / r_{s} \leq 2 \sqrt{3} / \sqrt{5}$. (b4) Body-centered lattice $\Lambda_{4-4}$ pattern when $2 \sqrt{3} / \sqrt{5} \leq r_{c} / r_{s}$.

sors are needed for global connectivity. Transmission of basic connectivity structure from "lines" or "planes" to "bodies" reflects transmission of constraints. When $r_{c}$ is small, connectivity constraints dominates. The structure follows the most efficient way to achieve required connectivity, e.g., "lines" in lattice $\Lambda_{2-1}$ pattern. When $r_{c}$ is large, coverage constraints dominates. The structure follows the most efficient way to achieve full coverage while connectivity is the byproduct. It is witnessed by the final convergence of patterns to lattice $\Lambda_{2-4}\left(\Lambda_{4-4}\right)$ pattern that is conjectured optimal in [8] for full coverage. Lattice $\Lambda_{2-2}$ and $\Lambda_{2-3}$ patterns illustrates the gradual shifting of constraints dominance from connectivity to coverage.

\section{PRACTICAL CONSIDERATIONS}

In this section, we discuss some practical issues beyond our mathematical abstraction.

- On the long path problem: As we discussed above, some extra nodes are needed at the volume boundaries for global connectivity in lattice $\Lambda_{2-1}, \Lambda_{2-2}, \Lambda_{4-1}$ and $\Lambda_{4-2}$ patterns. As shown in Fig. 7, ABCDEFGH is the volume where sensors are deployed. In Fig. 7(a), we show the case when lattice $\Lambda_{2-1}$ pattern is deployed. In this case, to achieve global connectivity, extra sensors need to be deployed at the boundary faces ADHE and BCGF. For each boundary faces, we first add sensors to connect "lines" in the lattice $\Lambda_{2-1}$ pattern, as illustrated by JK in Fig. 7(a), such that 2-connectivity are achieved in planes, for which we illustrate one by IJKL. Then we connect these parallel planes by adding sensors as illustrated by MN. The number of extra nodes needed in one boundary face is relatively small. For instance, when $r_{c}=r_{s}=30 \mathrm{~m}$, we need around 17,200 nodes to achieve 2-connectivity in a cube with edge length $1000 \mathrm{~m}$. while in each boundary face, we need around $1.7 \%$ (300) extra nodes. The possible long path problem can be overcome by adding these faces inside the deployment volume, as shown in Fig. 7(b). For $\Lambda_{4-1}$ and $\Lambda_{4-2}$, we have separately connected planes already rather than lines. So even smaller number of nodes are needed to construct a connection face.

- On Practical Sensing and Communication Models: In underwater networks, there is no well accepted model for sensing or communication along each possible 3D direction. In this paper, we consider practical models in aerial space.

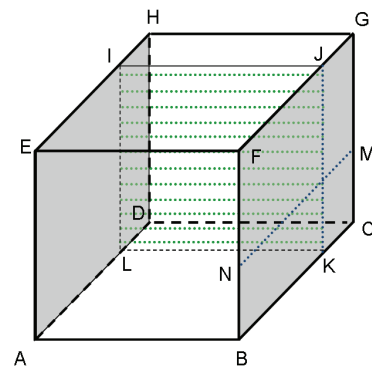

(a)

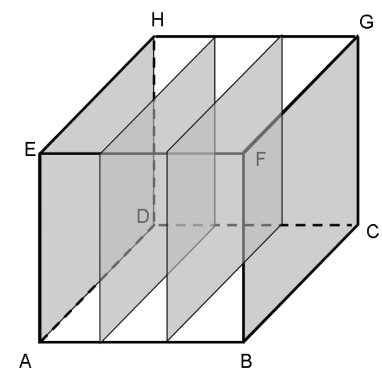

(b)
Figure 7: ABCDEFGH is the volume where sensors are deployed. (a) Lattice $\Lambda_{2-1}$ pattern is used. In the boundary face, JK is to connect all the "lines" in one plane, and NM is to connect all planes. (b) The long path problem can be overcome by adding such connection faces inside the target volume.

S. Megerian et al. in [27] propose that the quality of sensing gradually attenuates with increasing distance. Y. Zhou et al. in [28] propose a probabilistic sensing model where the detection probability changes for different target distance. When the above models are used, sensing sphere can still be obtained by setting a sensing range threshold that is decided by desirable sensing quality or detection probability. For some sensor types, the sensing capability may vary along different directions. One typical model obtained from real device experiments by Cao et al. in [29] suggests the sensing capability roughly follows Gaussian distribution over different directions. Denote the average sensing radius over all the directions by $\mu$ and the standard deviation by $\sigma^{2}$. In a particular direction, the probability for sensing range $X$ being $x$ is given by the following

$$
P\{X=x\}=\exp \left(-\frac{(x-\mu)^{2}}{2 \sigma^{2}}\right) /(\sigma \sqrt{2 \pi}) .
$$

As $\sigma$ increases, the sensing field is more close to a sphere. We study by simulation the impact from such sensing irregularity on coverage in lattice $\Lambda_{2}$ pattern. The results are shown in Fig. 8. We notice that higher sensing irregularity will result in lower overall coverage. When $r_{c} / r_{s} \geq 2 \sqrt{3} / 5$, the coverage will not change since the deployment pattern keeps 


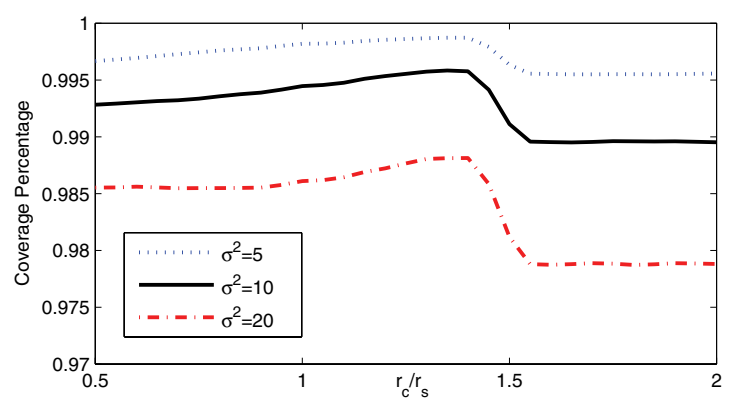

Figure 8: Sensors each with $\mu=30 \mathrm{~m}$ are deployed in a $1000^{3} \mathrm{~m}^{3}$ cube following the lattice $\Lambda_{2}$ pattern. The coverage in percentage is obtained by first generating $10^{6}$ points within the cube, and then checking how many percent of them are covered. Every value presented is the average over 100 times simulation.

same. One interesting observation is that there exists increment on coverage percentage from $r_{c} / r_{s}=0.5$ to $2 \sqrt[3]{3} / \sqrt{3}$. It illustrates that smaller $r_{c}$, which implies more overlapped area, does not always suggest better tolerance of sensing irregularity. Tolerance also depends on the pattern architecture. In lattice pattern $\Lambda_{2-1}$ and $\Lambda_{2-2}$, sensors are connected in "lines". Sensing irregularity along lines can be tolerated well. Lattice $\Lambda_{2-3}$ patterns can tolerate irregularity along all directions. But when $r_{c}$ further increases, the decreasing of overlapped area dominates the tolerance capability, which explains the rapid decrement of coverage percentage. The simulation results for 4-connectivity pattern generate the same observation. We do not present them due to the space limitations.

In reality, the communication wireless signal undergoes attenuation and various disruptive physical phenomena in the air. We consider a widely used model suggested by Zuniga and Krishnamachari in [30]. This model established the function of the distance between the transmitter and the receiver and the communication link quality measured by packet reception rate (PRR). PRR at distance $d$ can be expressed as

$$
\operatorname{PRR}(d)=\left(1-\frac{1}{2} \exp \left(-\frac{P_{t}-P L(d)-P_{n}}{2}\right)\right)^{8 \ell},
$$

where $P_{t}$ is the output power of the transmitter, $P L(d)$ is the path loss at distance $d, P_{n}$ is the noise floor and $\ell$ is the frame length. (Interested reader may refer to [30] for detailed derivation.) Under this model, we consider a connection established between two nodes only if the PRR from each other is above a certain threshold. By simulation, we investigate the effect from the above model on the probability for one sensor in lattice $\Lambda_{2}$ pattern to connect all 2 neighbors. The results are shown in Fig. 9. (The results for 4-connectivity lattice pattern are similar and not presented here due to the limitations of space.) We notice from Fig. 9 that that probability transition from 1 to 0 is sharp. This implies the connectivity will deteriorate fast when transmission power decreases in lattice $\Lambda_{2}$ pattern.

- On other practical settings: Individual sensors sometimes will not be exactly deployed at the precalculated locations due to deployment errors or boundary and location constraints. Denote the maximum random error by $\varepsilon$. In a

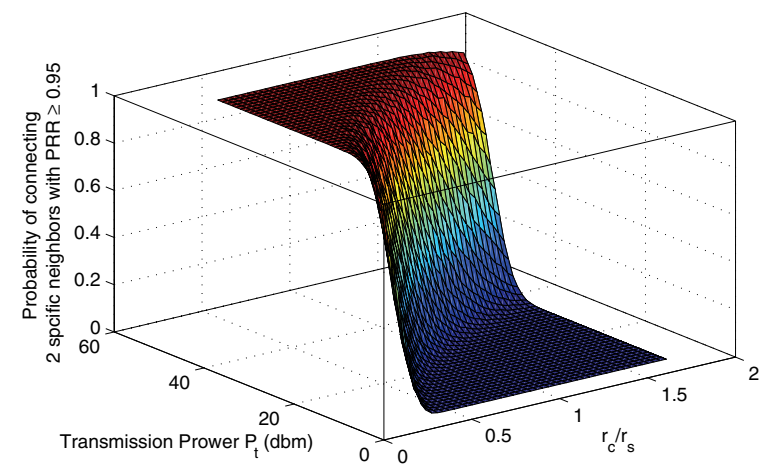

Figure 9: A connection is considered established when $P R R \geq 0.95 . r_{c} / r_{s}=0.3 \sim 1.8$. For each combination of $P_{t}$ and an optimal deployment pattern, we run simulation 10,000 times. The probability is then the ratio of number of times over 10,000 when a sensor can connect with 2 neighbors. Other parameters are from empirical data [30].

conservative way, to ensure the coverage and connectivity, we can use $r_{s}^{\prime}=r_{s}-\varepsilon$, and $r_{c}^{\prime}=r_{c}-\varepsilon$ to decide sensing and communication sphere and then the optimal pattern accordingly. Also, nodes in 3D spaces may not be homogenous. There can be certain powerful gateways routing data between sensors and the base station. In such cases, our optimal patterns are still valuable. In a simple example, we can consider the sensor to gateway communication range as $r_{s}$, and the gateway to gateway communication range as $r_{c}$. Ensuring that the whole network is fully covered (with coverage range $r_{s}$ ) as well as multiple-connected (with communication range $r_{c}$ ) means that each sensor in the network can communicate directly with at least one gateway and each gateway has multiple routing paths to other gateways. Fully studying these issues is part of our future research.

\section{CONCLUSION}

In this paper, we have designed a set of patterns for lowconnectivity $(k \leq 4)$ and full-coverage WSNs in 3D space. We have proved the optimality of 1 and 2 connectivity patterns under any value of the ratio of $r_{c} / r_{s}$ among the right lattice deployment patterns. We have investigated the evolutions among all the proposed low-connectivity patterns. The proposed patterns can save a significant number of sensor nodes, and provide insights for further pattern optimality exploration in 3D WSNs.

We are to explore the optimal deployment patterns to achieve full-coverage and multiple-connectivity in 3D space among more general regular patterns. Extending our research to more practical scenarios is also one of our future directions.

\section{ACKNOWLEDGEMENT}

This work was supported by the US National Science Foundation (NSF) CAREER Award CCF-0546668, the Army Research Office (ARO) under grant No. AMSRD-ACC-R 50521CI; High Technology Development Program and Natural Science Foundation of Guangdong \& Natural Science Foundation of P. R. China under Grant No. 2006B11201004, No. 
32207013 and No. 07005930; SAR Hong Kong RGC Competitive Earmarked Research Grant (CERG) No. 9041350 (CityU 114908), CityU Applied R \& D Centre (ARD(Ctr)) No. 9681001.

Any opinions, findings, conclusions, and recommendations in this paper are those of the authors and do not necessarily reflect the views of the funding agencies.

\section{REFERENCES}

[1] M. Campbell, "Intelligence in three dimensions: we live in a 3-d world, and so should computers," http://www.neptec.com/News2006/1Oct06-MilAero. html, 2006.

[2] M. K. Watfa, "Practical applications and connectivity: Algorithms in future wireless sensor networks," International Journal of Information Technolog, vol. 4, pp. 18-28, 2007.

[3] "Aerial common sensor (acs)," http://www.global-security.org/intell/systems/acs.htm, 2007.

[4] W. Tsujita, A. Yoshino, H. Ishida, T. Moriizumi, "Gas sensor network for air-pollution monitoring," Sensors and Actuators B: Chemical, Vol. 110, no. 2, pp. 304-311, 2005.

[5] J. Partan, J. Kurose, and B. N. Levine, "A survey of practical issues in underwater networks," in Proc. of the International Workshop on UnderWater Networks (WUWNet), 2006.

[6] J. Heidemann, W. Ye, J. Wills, A. Syed, and Y. Li, "Research challenges and applications for underwater sensor networking," in Proc. of IEEE Wireless Communications and Networking Conference (WCNC), 2006.

[7] W. Cheng, A.Teymorian, L. Ma, X. Cheng, X. Lu, and Z. Lu, "Underwater localization in sparse $3 \mathrm{~d}$ acoustic sensor networks," in Proc. of IEEE Annual Conference on Computer Communications (IEEE INFOCOM) , 2008.

[8] Thomson and S. W. L. Kelvin, "On the division of space with minimum partition area," Philosophical Magazine, no. 24, pp. 503-514, 1887.

[9] R. P. Bambah, "On lattice coverings by spheres," in Proc. of Nat. Sci. India, no. 10, pp. 25-52, 1954.

[10] E. S. Barnes, "The covering of space by spheres," Canad. J. Math., no. 8, pp. 293-304, 1956.

[11] L. Few, "Covering space by spheres," Mathematika, no. 3, pp. 136-139, 1956.

[12] R. P. Bambah, "Lattice coverings with four-dimensional spheres," in Proc. Cambridge Phil. Soc., no. 50, pp. 203-208, 1954.

[13] M. N. Bleicher, "Lattice coverings of n-space by spheres," Canad. J. Math., no. 14, pp. 632-650, 1962.

[14] H. S. M. Coxeter, Introduction to Geometry. New York: John Wiley, 1961.

[15] T. C. Hales, "A proof of the kepler conjecture," Annals of Mathematics, no. 162, pp. 1065-1185, 2005.

[16] N. Sloane, "The proof of the packing," Nature, vol. 425, pp. 126-127, 2003.

[17] G. G. Szpiro, Kepler's Conjecture:How Some of the Greatest Minds in History Helped Solve One of the Oldest Math Problems in the World. John Wiley Sons, Inc., 2003.
[18] X. Bai, S. Kumar, D. Xuan, Z. Yun, and T. H. Lai, "Deploying wireless sensors to achieve both coverage and connectivity," in Proc. of the ACM International Symposium on Mobile Ad Hoc Networking and Computing (ACM Mobihoc), 2006.

[19] X. Bai, Z. Yun, D. Xuan, T. H. Lai, and W. Jia, "Deploying four-connectivity and full-coverage wireless sensor networks," in Proc. of IEEE Annual Conference on Computer Communications (IEEE INFOCOM) , 2008.

[20] X. Bai, D. Xuan, Z. Yun, T. H. Lai, and W. Jia, "Complete optimal deployment patterns for full-coverage and k-connectivity $(\mathrm{k} \leq 6)$ wireless sensor networks," in Proc. ofthe ACM International Symposium on Mobile Ad Hoc Networking and Computing (ACM Mobihoc), 2008.

[21] S. M. N. Alam and Z. J. Haas, "Coverage and connectivity in three-dimensional networks," in Proc. of ACM International Conference on Mobile Computing and Networking (Mobicom), 2006.

[22] M. K. Watfa and S. Commuri, "A coverage algorithm in 3d wireless sensor networks," The 1st International Symposium on Wireless Pervasive Computing, 2006.

[23] C. F. Huang, Y. C. Tseng, and L. C. Lo, "The coverage problem in three-dimensional wireless sensor networks," in Proc. of IEEE Global Communications Conference (Globecom), 2004.

[24] H. Yan, Z. Shi, and J. Cui, "Dbr: Depth-based routing for underwater sensor networks," IFIP Networking, 2008.

[25] D. Pompili and T. Melodia, "Three-dimensional routing in underwater acoustic sensor networks," 2nd ACM international workshop on Performance evaluation of wireless ad hoc, sensor, and ubiquitous networks, 2005.

[26] Z. Zhou, J. Cui, and A. Bagtzoglou, "Scalable localization with mobility prediction for underwater sensor networks," in Proc. of IEEE Annual Conference on Computer Communications (IEEE INFOCOM), 2008.

[27] S. Megerian, F. Koushanfar, G. Qu, G. Veltri, and M. Potkonjak, "Exposure in wireless sensor networks: Theory and practical solutions," Wireless Networks, vol. 8, no. 5, pp. 443-454, 2002.

[28] Y. Zhou and K. Charkrabarty, "Sensor deployment and target localization based on virtual force," in Proc. of IEEE Annual Conference on Computer Communications (IEEE INFOCOM), 2003.

[29] Q. Cao, T. Yan, J. A. Stankovic, and T. F. Abdelzaher, "Analysis of target detection performance for wireless sensor networks," in Proc. of International Conference on Distributed Computing in Sensor Systems (DCOSS), 2005.

[30] M. Zuniga and B. Krishnamachari, "Analyzing the transitional region in low power wireless links," Technical Report 04-823, University of Southen California, 2004.

[31] X. Bai, C. Zhang, D. Xuan and W. Jia, "Full-Coverage and k-Connectivity $(k=14,6)$ Three Dimensional Networks," in Proc. of IEEE Annual Conference on Computer Communications (IEEE INFOCOM), 2009. 